\title{
Effect of stroke prevention medication on aortic atheroma progression assessed using new statistical paradigm
}

Pratyaydipta Rudra', Pranab K. Sen ${ }^{1}$, Lauren Dennis ${ }^{2}$ and Souvik Sen ${ }^{2 *}$

*Correspondence: Souvik.Sen@uscmed.sc.edu

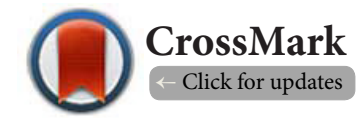

${ }^{1}$ Department of Biostatistics, University of North Carolina, Chapel Hill, North Carolina, USA.

${ }^{2}$ Department of Neurology, University of South Carolina School of Medicine, Columbia, South Carolina, USA.

\begin{abstract}
Background: Progression of aortic arch atheroma (AA) is associated with vascular events in patients with stroke or transient ischemic attack (TIA). Studies investigating effect of stroke prevention medication on AA progression are limited to post-cerebral segments that may not be related to stroke pathophysiology and do not use information from the relevant segments adjacent to the origin of the cerebral blood vessels.
\end{abstract}

Methods: AA atheroma was detected on baseline transesophageal echocardiogram (TEE) in 167 consecutive patients who had stroke or TIA. Of these, 125 consented to a follow-up TEE at12 months. Adequate paired AA images were obtained in 117 (78 with strokes, 39 with TIAs), which allowed detailed measurements of plaques. On admission for their index stroke or TIA, patients were assessed for stroke risk factors and stroke prevention medications (Statins, Antiplatelet therapy and oral anticoagulant). We assessed three segments of the AA adjacent to the origin of the cerebral blood vessels for plaque thickness by TEE and analyzed the effect of medication using joint modeling, with due consideration to confounding covariates. The statistical paradigm included a permutation testing procedure to determine the significance of medication effect on plaque progression in the three segments in combination.

Results: Using the specified statistical paradigm, it is evident that statin therapy is effective in arresting the AA progression; however, there is no significant change induced by anticoagulant therapy. The cleaned data, after removal of influential observations, shows even more significance for the statin therapy. The constrained hypothesis tests in both cases show a smaller p-value suggesting that the statin therapy may be effective in arresting the AA progression.

Conclusions: In this preliminary study of stroke/TIA patients with AA atheroma on transesophageal echocardiogram, AA atheroma progression was arrested by statin therapy, without any significant change induced by anticoagulant therapy. The statistical paradigm with due consideration of all segments implicated in stroke pathophysiology may be used to test the effect of other stroke prevention medications on AA progression.

Keywords: Aorta, atherosclerosis, disease progression, echocardiography, stroke

\section{Introduction}

Significant aortic arch atheroma (AA) is the second most prevalent risk for cardioembolic stroke after atrial fibrillation, present in $16-20 \%$ of stroke/TIA patients $[\mathbf{1 , 2}]$. It is a risk factor for new and recurrent stroke [4] and has lack of definitive treatment effect [5]. Among patients with AA, a subgroup that underwent progression AA in the ascending and arch segments were found to be independently associated with recurrent vascular events [6]. Retrograde diastolic blood flow in the proximal descending aorta, which connects significant plaques thickness with brain-supplying arteries, has been identified as a possible source of brain embolism [7]. In absence of 
randomized clinical trials, observational studies shed light on the effect of stroke prevention medications on AA measured at baseline. Most of the observational studies do not utilize the maximum information from the continuous measurement of plaque from all three segments of AA implicated in stroke pathophysiology [6]. Also, they do not explore the relationship of the treatment with the thickness of the plaque at different segments of the aorta in combination. Even when multiple segments were considered [6], the relationships are considered marginally, and therefore, the correlation structure among the thickness of plaque at different segments of the aorta, was not considered. Sometimes the information obtained was lost due to the use of a discrete grade scale or forming of class intervals. These limitations significantly limit the ability of clinicians to test the effect of stroke prevention medications on AA.

Recently, we conducted a prospective study using detailed serial-view, quantitative plaquethickness measurements andmethods to ensure imaging and gradation in similar locationsover a period of 12 months, to accurately estimate the rate ofprogression. We found that instroke/TIA patients with AA atheroma progression on repeat TEE at 12 months were more independently likely to experience recurrent vascular event [8]. No difference existed in theproportion of patients undergoing antiplatelet therapy, oralanticoagulation, or statin therapy. A limitation of the assessment of AA progression was the way the definition that included measures in the aortic arch segments only, eliminating the descending segments, and transformation of aortic arch plaque thickness from continuous variable to a clinically relevant grade. To counter these limitations we used the aortic plaque thickness in the ascending arch and descending segments as a continuous variable. Using an innovative statistical paradigm we investigated if the stroke prevention medications (statins, antiplatelet therapy, and oral anticoagulants) influenced AA progression and if the influence was independent of the stroke risk factors.

\section{Patients and methods}

Consecutive stroke/TIA patients $(n=307)$ underwent TEE within one month of symptom onset as a part of their stroke workup. All patients had computed tomography/MRI of the brain to confirm stroke and had risk factors assessed at admission for their index stroke/TIA. Exclusion criteria include age $<18$ years, intracerebral hemorrhage, subarachnoid hemorrhage, coma, conditions limiting life expectancy to $<12$ months (for example, end-stage cancer), and no aorticatheroma on baseline TEE. Of these patients, 167 had evidence of aortic atheroma (measurable plaque $\geq 1 \mathrm{~mm}$ in ascending aorta, arch,or descending thoracic aorta), and 125 of these eligible patients consented to a follow-up TEE at 12 months based on a protocol approved by the institutional review board. Of the 42 patients who did not consent, 35 refused and 7 were deceased before their follow-up TEE. Adequate paired aortic images were obtained in 117 of 125 patients ( 78 with stroke, 39 with TIA), which enabled us tomake detailed plaque measurements.

\section{TEE assessment of AA}

A comprehensive TEE with detailed imaging of the aorta was performed with a Hewlett-Packard 21364A omniplane probe (Hewlett-Packard, Palo Alta, CA). Imaging and quantification of AA atheroma were conducted with modifications of the previously described methods [2]. The aortic arch was definedas the portion of the aorta between the curve at the end of the ascendingaorta and the origin of the left subclavian artery. The descending thoracic aorta extended from the origin of the left subclavian artery to the level where the thoracic aorta crossed the diaphragm [2]. Briefly, the ascending and arch segments of the thoracic aorta were imaged at a probe depth of $\sim 30 \mathrm{~cm}$ with a multiplane angle of $100^{\circ}$ to $150^{\circ}$ to view the vessel in the long axis. The descending thoracic aorta was examined by advancing the probe to the distalesophagus, imaging the aorta in cross section (at $0^{\circ}$ ), and then slowly withdrawing the probe to image proximal segments. As the transducer reached the $A A$, the multiplane angle was rotated to between $0^{\circ}$ and $90^{\circ}$ to acquire sequential short-axis views.

The modifications included the acquisition of digital images of the diseased areas in each segment of the AA with annotation of the distance of the transducer from the incisors. Identical locations in the AA were evaluated on the 1 -year examination, with the depth of the transducer, plaque morphology, and surrounding anatomic landmarks used for guidance. Two observers, masked to clinical data and the order of the TEE, independently quantified atheroma. Plaque thickness was measured as the maximal thickness of the intimal and medial layers in the ascending, arch and descending segments. Progression of AA was assessed by the objective difference in plaque thickness in the ascending, arch and descending segments.

\section{Clinical definitions}

Stroke risk factors, stroke, and TIA were defined on the basis of previously described criteria. Stroke risk factors were assessed at the time of the initial qualifying event (stroke or TIA). Antiplatelet therapy included aspirin, aspirin with extended-release dipyridamole, clopidogrel, and ticlopidine. Oral anticoagulation implied Warfarin therapy for a known indication (for example, atrial fibrillation), and statin therapy indicated daily statin therapy. The medication history was collected at the time of the protocol-mandated 12-monthTEE examination.

\section{Statistical analysis}

The statistical analysis of the data demands the fitting of a multi-response model since the design involves measurement of plaque thickness at three points, the ascending, arch, and descending segments of the aorta, and they are likely to be inter-correlated. Further, plaque measurements have been 
made at 0 and 12 month/s (time-points) on the same subject where progression and regression may both occur over this period. We consider the difference in thickness between the two time points as our response, which is a three component vector. We use a multivariate regression model with such response variable, two treatments and five other covariates.

The primary predictors of interest are the binary indicators of the two medications - statin and anticoagulant therapy. Five covariates (Age, Hypertension, Diabetes, Hypercholesterolemia, Smoking) which are believed to be potential confounders are included in the model. Age is a continuous predictor, all others being binary indicators indicating the presence or absence of the trait.

The multivariate regression model can be mathematically written as

$$
Y_{i}=\beta X_{i}+\varepsilon
$$

Where $Y_{i}$ is a 3-component vector representing the response, $\beta$ is a $3 \times 7$ matrix of regression coefficients, $X_{i}$ is a $7 \times 1$ matrix representing the explanatory variables, and $\varepsilon_{i}^{\prime}$ s are independent and identically distributed 3-component random errors assumed to follow a $N_{3}(0, \Sigma)$ distribution, $i=1,2 \ldots . ., n$ (sample size).

If we want to test the effect of the a treatment, without loss of generality assuming it to be the first explanatory variable, the null hypothesis is

$$
H_{0}: \beta_{1}=0
$$

Where $\beta=\left(\beta_{1}, \beta_{2}\right)$ is a $3 \times 1$ and $3 \times 6$ partition of the coefficient matrix and 0 is the 3 -component zero vector.

\section{Initial processing of the data}

The data included 112 samples with no missing values. It was observed that the status of missingness was unrelated with both the response and the covariates. Thus, the data can be assumed to be missing completely at random. Usual MANOVA methods are applicable to this multi-response regression set up. However, visual inspection of the data indicated that the response may not be multivariate normal. Further statistical tests confirmed very significant deviation from multivariate normality. Transformations such as log transformation do not improve the situation. Also, the data includes some extremely influential observations that might bias the analysis. It is well known that MANOVA is very sensitive to influential observations. We identified seven such observations using methods to detect influential observations in multi-response regression [9]. Chi-square QQ-plot and the tests of multivariate normality reflect that the data deviates significantly from multivariate normal even after the removal of the influential observations.

\section{Analysis methods}

The Wilks' Lambda test (likelihood ratio test for MANOVA) requires the multivariate normality of the response. The Wilks' Lambda test statistic is defined as

$$
U=\frac{|E|}{|E+H|}
$$

where $\mathrm{H}$ is the hypothesis sum of squares and sum of products matrix and $E$ is the error sum of squares and sum of products matrix. Mathematically, the two sum of squares and sum of products matrices can be defined as

$$
\begin{gathered}
E=\frac{1}{n} \sum_{i=1}^{n}\left(Y_{i}-\hat{\beta} X_{i}\right)\left(Y_{i}-\hat{\beta} X_{i}\right)^{\prime} \\
H=\hat{\beta}_{1} A_{11.2} \hat{\beta}_{1} \\
A=\sum_{i=1}^{n} X_{i} X_{i}^{\prime}
\end{gathered}
$$

Is partitioned as $A=\left(\begin{array}{ll}\mathrm{A}_{11} & \mathrm{~A}_{12} \\ \mathrm{~A}_{21} & \mathrm{~A}_{22}\end{array}\right)$

Corresponding to the dimensions of $\beta_{1}$, and

$$
A_{11.2}=A_{11}-A_{12} A_{22}^{-1} A_{21}
$$

If the response is normal, the Wilks' Lambda statistic follows a Wilks' Lambda distribution. For large samples, Bartlett's approximation [10] is usually used to test where an appropriately adjusted logarithm of Wilks' Lambda statistic follows a chisquare distribution. In our case, the Bartlett approximation is given by

$$
-\left(n-7-\frac{3}{2}\right) \log (U) \sim \chi_{3 \mathrm{n}}^{2}
$$

However, these tests rely a lot on the assumed multi-normality of the response vectors. In clinical data, when such multinormality does not hold, the vector of the log-values of the responses is assumed to be multi-normally distributed. In this case, even the log transformed data does not appear to follow normality and the sensitiveness to the departure from this log-multi-normality assumption remains a genuine concern. We also tried more sophisticated and widely used transformations such as Box-Cox transformation [11], both marginally and jointly. However, the data still remained significantly different from multivariate normal after the transformation. We believe that the original data highly departs from multi-normality in a way that simply transforming the data cannot resolve.

Because of such significant departure from the multivariate normality, we propose a permutation test that can capture the true behavior of the tail of the distribution. We use the same Wilks' Lambda statistic defined in (1), but obtain the null distribution using permutation instead of using the Wilks' Lambda distribution. When testing the effect of a treatment, both the response and the treatment is adjusted for all the other covariates, and the residuals are used for testing. This approach is validated by the Frisch-Waugh-Lovell theorem $[12,13]$. Wilks' Lambda statistic is used as the test statistic, but a permutation test by permuting the treatments is used instead of the theoretical distribution of Wilks' Lambda. Such 
a permutation test only requires that the null hypothesis relates to some permutation invariance structure, and is not dependent on the underlying normality assumption. When testing for a given treatment, the values of the variable corresponding to that treatment (residualized) is permuted keeping the response vector (residualized) fixed. 10000 permutations are used. Clearly, the null hypothesis assuming the absence of that treatment effect is invariant with respect to such permutation. Figure 4 shows that the tail of the Wilks' Lambda theoretical distribution is heavier than the distribution of the test statistic obtained by permutation.

It is well known that the stroke risk is mostly affected by the thickness of plaque at the aortic arch. This fact motivated us to also carry out the hypothesis test for a constrained alternative hypothesis where the alternative hypothesis assumes that the treatment reduces the thickness of plaque at the aortic arch. The likelihood ratio test (LRT) statistic for such a constrained hypothesis testing problem follows a chi-barsquare distribution under the null if normality is assumed [14]. Since the data deviates from normality, we used the same LRT statistic for the constrained alternative, but found out the null distribution using permutations as before.

\section{Results}

Of 125 eligible patients, 112 patients with no missing observations were considered for the analysis. After removal of influential observations, the data contains 105 samples. Tables 1,2 and Figure 1 show the clinical and demographic characteristics of the patients and the distribution for different treatments. A significant deviation from normality was noted in the 112 observations without missing observation (Table 3 ) as well as the 105 observations with the influential observations removed (Table 4). This deviation is further confirmed on the chi-square QQ-plot for testing multivariate normality for the 112 observations without missing observation (Figure 2) as well as the 105 observations with the influential observations

Table 1. Showing the summary of patient demographics and clinical characteristics.

\begin{tabular}{lll}
\hline & n & $\begin{array}{l}\text { Prevalence, \% or } \\
\text { value, Mean } \pm \text { SD }\end{array}$ \\
\hline Age & 112 & $65.5 \pm 11.3$ \\
Gender (Male) & 61 & $54.5 \%$ \\
Race (African American) & 85 & $75.9 \%$ \\
Hypertension & 90 & $80.4 \%$ \\
Diabetes & 29 & $25.9 \%$ \\
Hypercholesterolemia & 75 & $67 \%$ \\
Smoking & 25 & $22.3 \%$ \\
\hline
\end{tabular}

Table 2. Showing the cross classification of number of patients under different treatments.

\begin{tabular}{lll}
\hline & Statin & No Statin \\
\hline Anticoagulant & 17 & 12 \\
Antiplatelet & 48 & 35 \\
\hline
\end{tabular}

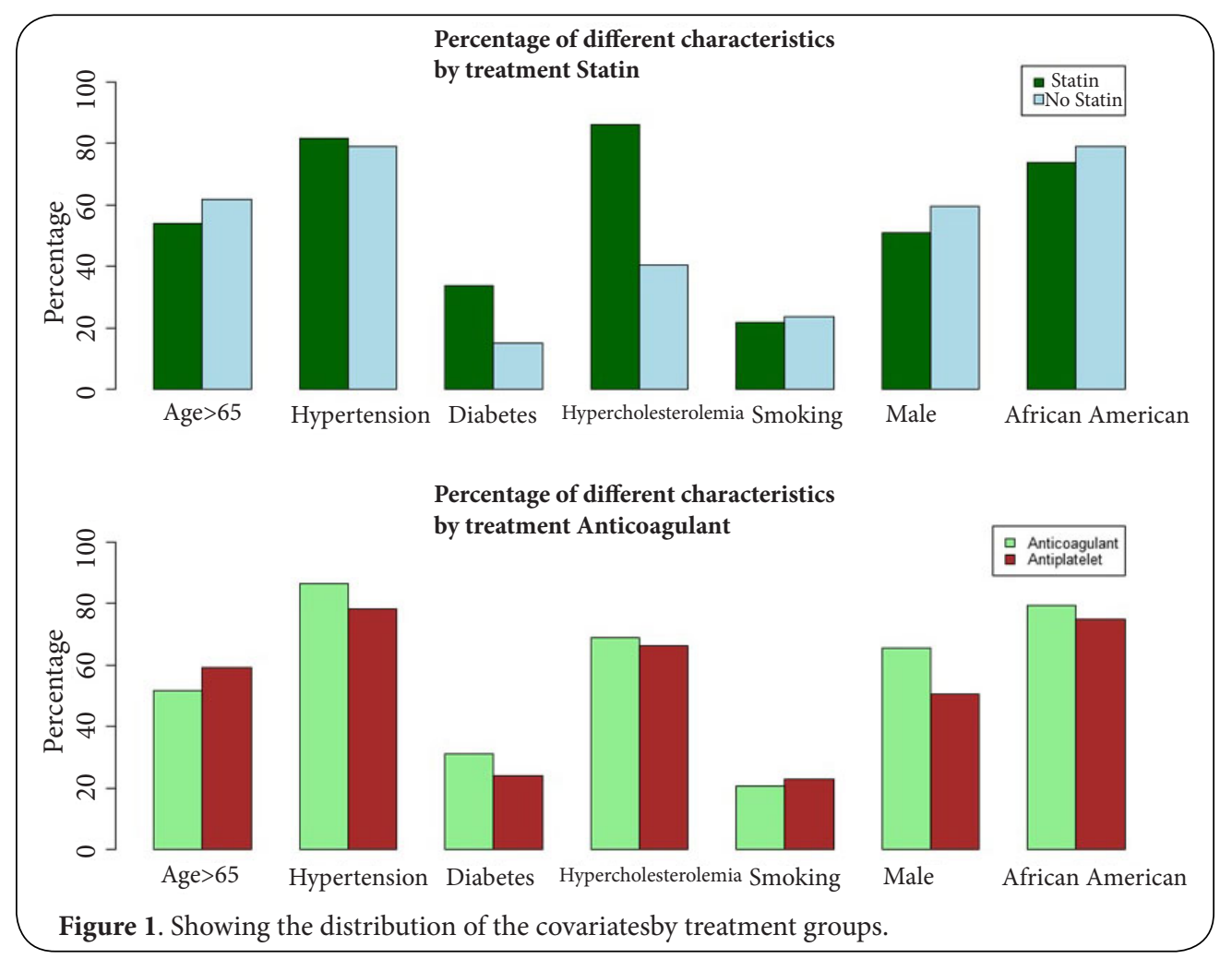


Rudra et al. Journal of Medical Statistics and Informatics 2016,

Table 3. Showing the tests of multivariate normality for the data (112 samples).

\begin{tabular}{ll}
\hline Test & P-value \\
\hline Mardia's test of Skewness & $2.16 \times 10^{-10}$ \\
Henze-Zirkler's Multivariate Normality Test & 0 \\
Royston's Multivariate Normality Test & $1.40 \times 10^{-18}$ \\
\hline
\end{tabular}

Table 4. Showing the tests of multivariate normality for the cleaned data (105 samples).

\begin{tabular}{ll}
\hline Test & P-value \\
\hline Mardia's test of Skewness & $5.88 \times 10^{-9}$ \\
Henze-Zirkler's Multivariate Normality Test & 0 \\
Royston's Multivariate Normality Test & $8.25 \times 10^{-17}$ \\
\hline
\end{tabular}

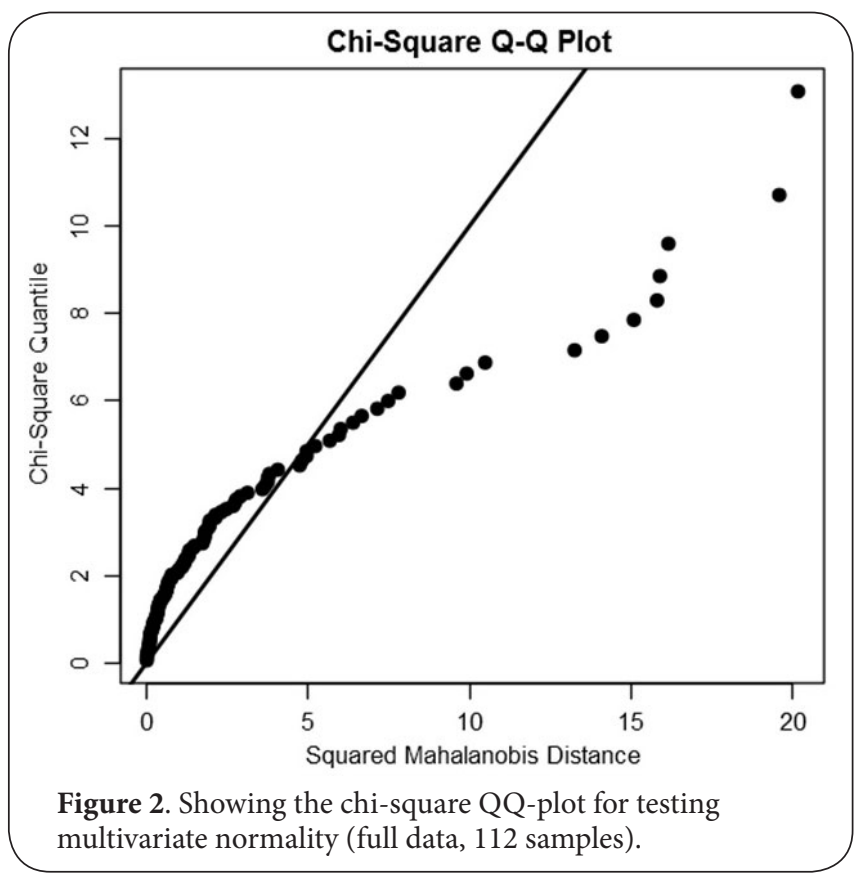

removed (Figure 3). If the data are multivariate normal, the squared Mahalanobis distance calculated from the residuals will follow a chi-square distribution with 3 degrees of freedom. The QQ-plot shows the quantiles of the observed squared Mahalanobis distance against the expected quantiles. Since many of points in the plotdeviate from the $y=x$ line, the data must be deviating from multivariate normal.

Because the data significantly deviates from the multivariate normality, we propose a permutation test (10000 permutations used) that can capture the true behavior of the tail of the distribution. Table 5 shows the results of the tests for the full data (112 samples). The $p$-values corresponding to the main effects of statin and anticoagulant therapy using the standard Wilks' lambda test for the multivariate regression model suggest a marginally significant effect of statin and non-significant effect of anticoagulant therapy compared
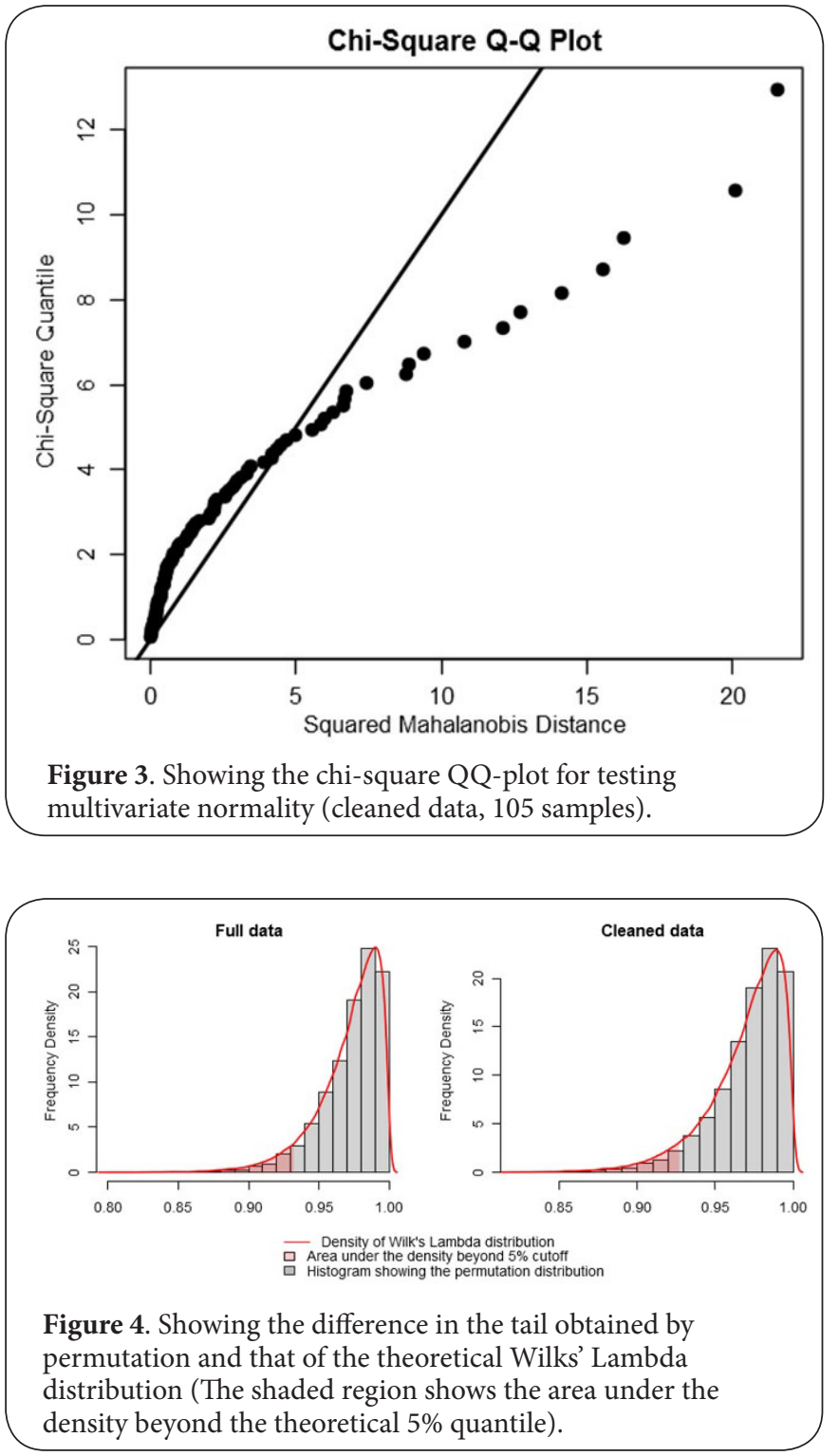

Table 5. Results of the hypothesis testing for the treatment effects (Full data, 112 samples).

\begin{tabular}{llll}
\hline Treatment & $\begin{array}{l}\text { Wilks' Lambda } \\
\text { test assuming } \\
\text { normality }\end{array}$ & $\begin{array}{l}\text { Permutation } \\
\text { test }\end{array}$ & $\begin{array}{l}\text { Constrained } \\
\text { permutation } \\
\text { test to test the } \\
\text { regression of } \\
\text { plaque }\end{array}$ \\
\hline Statin & P-value $=0.0449$ & P-value $=0.0310$ & P-value $=0.0253$ \\
Anticoagulant & P-value $=0.4517$ & P-value $=0.4222$ & P-value $=0.3592$ \\
\hline
\end{tabular}

with antiplatelet therapy. All the interaction effects were found to be non-significant. The second column of Table 5 depicts the $p$-values using the same Wilks' lambda statistic, but using permutation method to determine the null distribution. Finally, the third column shows the $p$-values for the constrained test against the alternative that the treatments 
result in a regression in the thickness of plaque at the aortic arch. It is evident that the statin treatment is effective in arresting the $A A$ progression, but there is no significant change when anticoagulant therapy is applied. It is also clear that our permutation testing method reveals the true significance of the statin therapy while the usual LRT, having a thicker tail under the null, shows only marginal significance. As expected, the constrained tests show a smaller $p$-value. The cleaned data after removal of influential observations shows even more significance for the statin therapy (Table 6). The constrained hypothesis tests in both cases show a smaller $p$-value for statin indicating that the statin therapy is indeed effective in arresting the $A A$ progression.

Table 6. Results of the hypothesis testing for the treatment effects (Cleaned data, 105 samples).

\begin{tabular}{llll}
\hline Treatment & $\begin{array}{l}\text { Wilks' Lambda } \\
\text { test assuming } \\
\text { normality }\end{array}$ & $\begin{array}{l}\text { Permutation } \\
\text { test }\end{array}$ & $\begin{array}{l}\text { Constrained } \\
\text { permutation } \\
\text { test to test the } \\
\text { regression of } \\
\text { plaque }\end{array}$ \\
\hline Statin & P-value $=0.0193$ & P-value $=0.0144$ & P-value $=0.0136$ \\
Anticoagulant & P-value $=0.5464$ & P-value $=0.5159$ & P-value $=0.4571$ \\
\hline
\end{tabular}

Figure 4 shows the histogram of the permutation distribution and the density of the corresponding Wilks' lambda distribution in the same plot (Panel 1: Full data, Panel 2: Cleaned data). The comparison of the tails of the two distributions indicates that the permutation test can reveal true significance because it has a lighter tail, and this further demonstrates the usefulness of our approach.

\section{Discussion}

In this preliminary study of stroke/TIA patients with AA atheroma on transesophageal echocardiogram, using astatistical paradigm with due consideration of all segments implicated in stroke pathophysiology, we are able to demonstrate that AA atheroma progression may be arrested by statin therapy, without any significant change induced by anticoagulant therapy. The effect of statin therapy on AA progression is in agreement with MRI based studies investigating atherosclerotic plaque in the thoracic and abdominal aorta. A small study of 27 patients measured plaque area and volume, using combined surface and transesophageal MRI, demonstrated that six months of statin therapy resulted in significant AA regression that was strongly associated with low-density lipoprotein (LDL) cholesterol reduction [18]. Two prior randomized studies of low-dose and higher-dose statin in patients with aortic and/or carotid plaques showed similar results. Significant regression in plaque was seen on MRI during therapy in one study and was related to LDL cholesterol but not to the statin dosage [19]. In the other study, this regression was related to LDL lowering and the statin dosage [20]. A recent open- labeled randomized trial tested the effect of 20-mg versus 5 -mg atorvastatin ( $1=\mathrm{N} 2=18$ ) on asymptomatic thoracic and abdominal aortic plaque measured by MRI, demonstrated a significant plaque regression over 2 years [21]. A more recent open-labeled randomized trial tested the effect of intensive $(\mathrm{N}=31)$ versus standard $(\mathrm{N}=29)$ rosuvastatin therapy based on LDL reduction also in asymptomatic thoracic and abdominal aortic plaque measured by MRI, demonstrated a significant plaque regression over one year [22]. While it seems likely that statin therapy may decrease stroke risk, the association between $\mathrm{AA}$ plaque regression and stroke risk reduction has never been demonstrated. Also, the results of such observational studies must be interpreted with caution as these studies investigated thoracic and abdominal aorta distal to the origin of cerebral blood vessels and to date no study has investigated the effect of stroke prevention medication on AA progression specific to segments that are adjacent to the origin of the cerebral blood vessels.

Recent guidelines on thoracic aortic disease recommend oral anticoagulation therapy with warfarin (INR 2.0 to 3.0) or antiplatelet therapy to be considered instroke patients with aortic arch atheroma $4.0 \mathrm{~mm}$ orgreater to prevent recurrent stroke (Class Ilb, level of evidence C) [23]. The data supporting this recommendation are derived from three observational studies suggesting that oral anticoagulation with Warfarin is not harmful in patients with aortic arch plaque, and there were fewer strokes in the patients who were given Warfarin by their physicians compared with those given antiplatelet therapy [24]. Although we have shown that AA progression is an independent predictor of recurrent vascular event in stroke/TIA patients, there is lack of evidence linking anticoagulants and/or antiplatelet therapy and AA progression. A recent prospective randomized controlled, open-labeled trial with blinded end point evaluation of patients with an ischemic stroke and aortic arch plaques $\geq 4 \mathrm{~mm}$ treated with combination antiplatelet therapy,was in conclusive, showing a non-significant $24 \%$ reduction in the rate of recurrent stroke, myocardial infarction, peripheralembolism, and vascular death (adjusted $\mathrm{P}=0.5$ ) [25]. These results are in agreement with ours as the results did not show anticoagulant or antiplatelet therapy to significantly influence recurrent vascular events in stroke/TIA patient.

The analysis models the three segments jointly instead of testing their marginal relationship with the treatment. Such joint modeling not only makes the procedure more valid, it may also increase the power. We have used a permutation testing procedure that is valid despite the data having a large departure from normality. Because the MANOVA tests have increased sensitivity to the assumption of normality, even slight departure from normality may result in incorrect conclusions if the usual Wilks' Lambda test is used. The use of permutation testing solves this problem by accurately approximating the tail probability of the null distribution. We also proposed a one-tailed test in the expected direction which results in 
higher significance. Sophisticated techniques of constrained statistical inference were used for this purpose. Therefore, we claim that our method is more robust as well as more powerful in detecting the significance of the treatment effect.

We have performed two tests: (i) statin vs no statin and (ii) anticoagulant vs antiplatelet. We have presented the $p$ values uncorrected for multiple hypothesis tests. Although the multiple testing correction procedures reduce the chance of accidental false discoveries, they often lead to very high type II error rate [15]. As suggested by Rothman [16], such adjustments are unnecessary if a few planned comparisons are made when there is a strong reason to believe that the alternative hypotheses might be true. This situation is clearly different from performing several tests in large datasets where we expect the null hypothesis to be true in most cases, in which case multiple testing adjustments must be used. Following this argument $[16,17]$, we feel multiple testing adjustments unnecessary for our analysis. However, we clearly mention that the $p$-values presented are un-adjusted, and the chance of making a false discovery is $5 \%$ for both the tests. If one wants to control the family-wise error rate, such adjustments has to be used; for example, Bonferroni adjusted $p$-values for statin will be not significant for the full data, but they will still be significant for the cleaned data.

There are few limitations to our study results. Due to the retrospective nature of our data analysis from a prospective observational cohort study, the result should be interpreted with caution. The study defines the specific statistical method to be employed in future prospective randomized trial to test the effect of medications on AA progression an independent risk factor for recurrent vascular events in stroke/TIA patients. The study results may not be equated to those obtained from a placebo controlled randomized clinical trial, and natural age related $A A$ atheroma progression indeterminable from this study. Since standard of care, in secondary stroke prevention include the treatment studied-statin therapy, antiplatelet and anticoagulant therapies [26], placebo controlled trial may be regarded as unethical and clinically impractical, retrospective analysis the only recourse to measure treatment effects. In this preliminary study of stroke/TIA patients with AA atheroma on transesophageal echocardiogram, AA atheroma progression was arrested by statin therapy, without any significant change induced by anticoagulant therapy. The statistical paradigm, with due consideration of all segments implicated in stroke pathophysiology, may be used to test the effect of other stroke prevention medications on AA progression. The methodology needs to be further validated in other similar clinical scenario with similar data limitations.

\section{Competing interests}

The authors declare that they have no competing interests.
Authors' contributions

\begin{tabular}{|l|c|c|c|c|}
\hline Authors' contributions & PR & PKS & LD & SS \\
\hline Research concept and design & $\checkmark$ & $\checkmark$ & -- & $\checkmark$ \\
\hline Collection and/or assembly of data & -- & -- & -- & $\checkmark$ \\
\hline Data analysis and interpretation & $\checkmark$ & $\checkmark$ & -- & $\checkmark$ \\
\hline Writing the article & $\checkmark$ & $\checkmark$ & $\checkmark$ & $\checkmark$ \\
\hline Critical revision of the article & $\checkmark$ & $\checkmark$ & $\checkmark$ & $\checkmark$ \\
\hline Final approval of article & $\checkmark$ & $\checkmark$ & $\checkmark$ & $\checkmark$ \\
\hline Statistical analysis & $\checkmark$ & $\checkmark$ & -- & $\checkmark$ \\
\hline
\end{tabular}

Acknowledgement and funding

Dr. Sen was funded by a clinical investigator development award from the National Institute for Neurological Disorders and Stroke, a division of the National Institutes of Health. Funding for the study was provided by National Institutes of Health grants 1K23NS02117 and RR00046.

\section{Publication history}

Editors: Xiang-Yang Lou, University of Alabama at Birmingham, USA. Feng Gao, Washington University School of Medicine, USA. Received: 22-Jan-2016 Final Revised: 24-Feb-2016

Accepted: 29-Feb-2016 Published: 11-Mar-2016

\section{References}

1. Cheitlin MD, Armstrong WF, Aurigemma GP, Beller GA, Bierman FZ, Davis JL, Douglas PS, Faxon DP, Gillam LD, Kimball TR, Kussmaul WG, Pearlman AS, Philbrick JT, Rakowski H, Thys DM, Antman EM, Smith SC, Jr., Alpert JS, Gregoratos G, Anderson JL, Hiratzka LF, Hunt SA, Fuster V, Jacobs AK, Gibbons RJ and Russell RO. ACC/AHA/ASE 2003 Guideline Update for the Clinical Application of Echocardiography: summary article. A report of the American College of Cardiology/American Heart Association Task Force on Practice Guidelines (ACC/AHA/ASE Committee to Update the 1997 Guidelines for the Clinical Application of Echocardiography). J Am Soc Echocardiogr. 2003; 16:1091-110. | Article | PubMed

2. Sen S, Wu K, McNamara R, Lima J, Piantadosi S and Oppenheimer SM. Distribution, severity and risk factors for aortic atherosclerosis in cerebral ischemia. Cerebrovasc Dis. 2000; 10:102-9. | Article I PubMed

3. Amarenco P, Cohen A, Tzourio C, Bertrand B, Hommel M, Besson G, Chauvel C, Touboul PJ and Bousser MG. Atherosclerotic disease of the aortic arch and the risk of ischemic stroke. N Engl J Med. 1994; 331:1474-9. | Article | PubMed

4. Atherosclerotic disease of the aortic arch as a risk factor for recurrent ischemic stroke. The French Study of Aortic Plaques in Stroke Group. N Engl J Med. 1996; 334:1216-21. I Article I PubMed

5. Tunick PA and Kronzon I. Atheromas of the thoracic aorta: clinical and therapeutic update. J Am Coll Cardiol. 2000; 35:545-54. | Article | PubMed

6. Sen S. Aortic arch plaque in stroke. Curr Cardiol Rep. 2009; 11:28-35. | Article I PubMed

7. Wehrum T, Kams M, Strecker C, Dragonu I, Gunther F, Geibel A, Drexl J, Hennemuth A, Schumacher M, Jung B and Harloff A. Prevalence of potential retrograde embolization pathways in the proximal descending aorta in stroke patients and controls. Cerebrovasc Dis. 2014; 38:410-7. | Article I PubMed

8. Sen S, Hinderliter A, Sen PK, Simmons J, LeGrys VA, Beck J, Offenbacher S, Moss K and Oppenheimer SM. Association of leukocyte count with progression of aortic atheroma in stroke/transient ischemic attack patients. Stroke. 2007; 38:2900-5. | Article | PubMed

9. Barrett, Bruce E and Robert F. Ling. General classes of influence measures for multivariate regression. Journal of the American Statistical Association. 1992; 87:184-191. | Article

10. Bartlett and Maurice Stevenson. A note on the multiplying factors for various $\chi \mathbf{2}$ approximations. Journal of the Royal Statistical Society. Series $B$ (Methodological). 1954; 296-298. 
Rudra et al. Journal of Medical Statistics and Informatics 2016,

11. Box, George EP and David R. Cox. An analysis of transformations. Journal of the Royal Statistical Society. Series B (Methodological). 1964; 211-252.

12. Frisch, Ragnar and Frederick V. Waugh. Partial time regressions as compared with individual trends. Econometrica: Journal of the Econometric Society. 1933; 387-401. I Pdf

13. Lovell, Michael C. Seasonal adjustment of economic time series and multiple regression analysis. Journal of the American Statistical Association. 1963; 58:993-1010. | Article

14. Silvapulle, Mervyn J and Pranab Kumar Sen. Constrained statistical inference: Order, inequality, and shape constraints. John Wiley \& Sons. 2011.

15. Perneger TV. What's wrong with Bonferroni adjustments. BMJ. 1998; 316:1236-8. | Article | PubMed Abstract | PubMed FullText

16. Rothman KJ. No adjustments are needed for multiple comparisons Epidemiology. 1990; 1:43-6. I Article I PubMed

17. Bender R and Lange S. Adjusting for multiple testing--when and how? J Clin Epidemiol. 2001; 54:343-9. | Article | PubMed

18. Lima JA, Desai MY, Steen H, Warren WP, Gautam S and Lai S. Statininduced cholesterol lowering and plaque regression after 6 months of magnetic resonance imaging-monitored therapy. Circulation. 2004; 110:2336-41. | Article | PubMed

19. Corti R, Fuster V, Fayad ZA, Worthley SG, Helft G, Chaplin WF, Muntwyler J, Viles-Gonzalez JF, Weinberger J, Smith DA, Mizsei G and Badimon $J$ J. Effects of aggressive versus conventional lipid-lowering therapy by simvastatin on human atherosclerotic lesions: a prospective, randomized, double-blind trial with high-resolution magnetic resonance imaging. J Am Coll Cardiol. 2005; 46:106-12. | Article | PubMed

20. Tunick PA, Nayar AC, Goodkin GM, Mirchandani S, Francescone S, Rosenzweig BP, Freedberg RS, Katz ES, Applebaum RM and Kronzon I. Effect of treatment on the incidence of stroke and other emboli in $\mathbf{5 1 9}$ patients with severe thoracic aortic plaque. Am J Cardiol. 2002; 90:13205. | Article | PubMed

21. Yonemura A, Momiyama Y, Fayad ZA, Ayaori M, Ohmori R, Kihara T, Tanaka N, Nakaya K, Ogura M, Taniguchi H, Kusuhara M, Nagata M, Nakamura H, Tamai S and Ohsuzu F. Effect of lipid-lowering therapy with atorvastatin on atherosclerotic aortic plaques: a 2-year follow-up by noninvasive MRI. Eur J Cardiovasc Prev Rehabil. 2009; 16:222-8. | Article I PubMed

22. Yogo $M$, Sasaki $M$, Ayaori $M$, Kihara $T$, Sato $H$, Takiguchi $S$, Uto-Kondo H, Yakushiji E, Nakaya K, Komatsu T, Momiyama Y, Nagata M, Mochio S, Iguchi $Y$ and Ikewaki K. Intensive lipid lowering therapy with titrated rosuvastatin yields greater atherosclerotic aortic plaque regression: Serial magnetic resonance imaging observations from RAPID study. Atherosclerosis. 2014; 232:31-9. | Article | PubMed

23. Hiratzka LF, Bakris GL, Beckman JA, Bersin RM, Carr VF, Casey DE, Jr., Eagle KA, Hermann LK, Isselbacher EM, Kazerooni EA, Kouchoukos NT, Lytle BW, Milewicz DM, Reich DL, Sen S, Shinn JA, Svensson LG and Williams DM. 2010 ACCF/AHA/AATS/ACR/ASA/SCA/SCAI/SIR/ STS/SVM guidelines for the diagnosis and management of patients with Thoracic Aortic Disease: a report of the American College of Cardiology Foundation/American Heart Association Task Force on Practice Guidelines, American Association for Thoracic Surgery, American College of Radiology, American Stroke Association, Society of Cardiovascular Anesthesiologists, Society for Cardiovascular Angiography and Interventions, Society of Interventional Radiology, Society of Thoracic Surgeons, and Society for Vascular Medicine. Circulation. 2010; 121:e266-369. | Article | PubMed

24. Sen S. Aortic arch plaque in stroke. Curr Cardiol Rep. 2009; 11:28-35. | Article | PubMed

25. Amarenco P, Davis S, Jones EF, Cohen AA, Heiss WD, Kaste M, Laouenan $C$, Young D, Macleod $M$ and Donnan GA. Clopidogrel plus aspirin versus warfarin in patients with stroke and aortic arch plaques. Stroke. 2014; 45:1248-57. | Article | PubMed

26. Kernan WN, Ovbiagele B, Black HR, Bravata DM, Chimowitz MI, Ezekowitz MD, Fang MC, Fisher M, Furie KL, Heck DV, Johnston SC, Kasner SE, Kittner SJ, Mitchell PH, Rich MW, Richardson D, Schwamm LH and Wilson JA. Guidelines for the prevention of stroke in patients with stroke and transient ischemic attack: a guideline for healthcare professionals from the American Heart Association/American Stroke Association. Stroke. 2014; 45:2160-236. | Article | PubMed

\section{Citation:}

Rudra P, Sen PK, Dennis L and Sen S. Effect of stroke prevention medication on aortic atheroma progression assessed using new statistical paradigm. J Med Stat Inform. 2016; 4:4.

http://dx.doi.org/10.7243/2053-7662-4-4 